\title{
Ensemble Kalman and Particle Filter for Noise-Driven Oscillatory Systems
}

\author{
Mohammad Khalil *, Abhijit Sarkar ${ }^{\dagger}$ and S Adhikari ${ }^{\ddagger}$
}

\begin{abstract}
Combined state and parameter estimation of dynamical systems plays an important role in many branches of applied science and engineering. A wide variety of methods have been developed to tackle the joint state and parameter estimation problem. The Extended Kalman Filter (EKF) method is a popular approach which combines the traditional Kalman filtering and linearisation techniques to effectively tackle weakly nonlinear and non-Gaussian problems. Its mathematical formulation is based on the assumption that the probability density function (PDF) of the state vector can be reasonably approximated to be Gaussian. Recent investigations have been focused on Monte Carlo based sampling algorithms in dealing with strongly nonlinear and non-Gaussian models. Of particular interest is the Ensemble Kalman Filter (EnKF) and the Particle Filter (PF). These methods are robust in handling general forms of nonlinearities and non-Gaussian models, albeit with higher computational costs. In this paper we report the joint state and parameter estimation of noise-driven oscillatory systems undergoing limit cycle oscillation using EKF, EnKF and PF.
\end{abstract}

\section{Introduction}

A wide variety of methods have been developed to tackle the joint state and parameter estimation of dynamical systems in the data assimilation research community. ${ }^{1-3}$ Due to its mathematical simplicity, Kalman Filter (KF) has gained immense popularity for optimal state estimation problems in linear systems with additive Gaussian noise. ${ }^{4} \mathrm{KF}$ results in a recursive analytical solution of the posterior probability distribution function (PDF) of the system state.

In the framework of filtering theory applied to parameter estimation, one or more unknown system parameters are appended to the original state vector. ${ }^{1,2}$ In general, the augmented state vector evolves nonlinearly regardless of whether the original system model is linear or nonlinear. This fact precludes the direct application of KF. Under certain conditions however, KF can be extended to the nonlinear case by linearising the model and measurement operators around the current estimate of the state vector, leading to the popular (but no longer optimal) Extended Kalman Filter (EKF). ${ }^{1,5}$ The major limitation of KF and EKF is the assumption of Gaussian

\footnotetext{
${ }^{*}$ Graduate Student, Department of Civil and Environmental Engineering, Carleton University, Mackenzie Building, Colonel By Drive, Ottawa, Ontario K1S 5B6, Canada.

${ }^{\dagger}$ Assistant Professor and Canada Research Chair, Department of Civil and Environmental Engineering, Carleton University, Mackenzie Building, Colonel By Drive, Ottawa, Ontario K1S 5B6, Canada.

${ }^{\ddagger}$ Chair of Aerospace Engineering, School of Engineering, University of Wales, Swansea, Singleton Park, Swansea SA2 8PP, UK, AIAA Senior Member.
} 
state vector which may produce instabilities and even divergence of the filter estimates, when applied to strongly nonlinear systems. ${ }^{6}$

Recently, Monte Carlo based sequential filtering algorithms have been developed to tackle the general case of nonlinear systems perturbed by non-Gaussian noise. Monte Carlo based approach involves representing the probability density of the state estimate by a finite number of randomly generated system states. Examples of Monte Carlo based filters include the Ensemble Kalman Filter (EnKF); ${ }^{1}$ and (ii) the Particle Filter $(\mathrm{PF}) .^{7}$

The usefulness of EnKF has been successfully demonstrated for different applications in data assimilation problem. ${ }^{1}$ EnKF inherits the same analysis step from KF. Subsequently, the ensemble members are propagated in time using the original nonlinear model operator. The measurement error covariance matrix in EnKF is estimated statistically from the ensemble. ${ }^{1}$ EnKF resolves the issue of poor error covariance evolution associated with EKF in many applications. ${ }^{1}$ In the context of structural dynamics, the EnKF has recently been introduced as a non-parametric identification tool by Ghanem and Ferro. ${ }^{8}$

The Gaussian assumption in EnKF greatly simplifies the analysis step. Although this approach to parameter estimation has been widely available in the literature, some publications reported rather poor performance by EnKF in certain applications. ${ }^{9}$ A more general approach, namely PF, is developed in the framework of Bayesian inference. ${ }^{7}$ This methodology relaxes the assumption of Gaussian posterior PDF and linearity of model and measurement operators. In this approach, the ensemble members (so-called particles) are properly weighted based on some metric relating to the observational data. In contrast to EnKF, the trajectories of the particles evolve independently without any analysis step (as detailed later). This fact partly undermines the efficacy of PF mandating larger ensembles incurring higher computational costs. This problem is partly alleviated using the so-called resampling technique. ${ }^{7}$ In the context of nonlinear dynamics, Manohar and Roy ${ }^{10}$ applied PF to identify a nonlinear stiffness parameter of a Duffing oscillator. ${ }^{11,12}$ To improve the performance of PF, hybrid filtering algorithms which combine EnKF with PF have also been proposed. ${ }^{13}$

In this paper, an extensive numerical experimentation is conducted to assess the performance of EKF, EnKF and PF in joint state and parameter estimation for Limit Cycle Oscillation (LCO). In particular, the attention is focused on tracking LCO of a Duffing oscillator perturbed by random noise. To the authors' best knowledge, such comparative study has not been widely reported in the literature of structural dynamics. The paper is organized as follows: A brief introduction to the EnKF is presented in Section II. In Section III, the formulation of the PF is reviewed. Section IV reports the results from a numerical example elucidating the capability of various filtering techniques. The paper concludes in Section $\mathrm{V}$ where a summary and findings of the current investigation are detailed.

\section{The Ensemble Kalman Filter}

EnKF, an extension of traditional KF for strongly nonlinear and non-Gaussian problems, is based on the representation of the probability density of the state estimate by a finite number (say $N$ ) of randomly generated system states (also called ensemble members). ${ }^{1}$ In this approach, the estimates of the state vector and the associated error covariance matrix are statistically estimated from the ensemble. As the observational data is assimilated using standard $\mathrm{KF}$ analysis step, each sample is then integrated forward in time independently using the full nonlinear evolution model. 
Consider a general representation of the evolution and measurement equations: ${ }^{7,10,14}$

$$
\begin{aligned}
& \mathbf{x}_{k+1}=\mathbf{g}_{k}\left(\mathbf{x}_{k}, \mathbf{f}_{k}, \mathbf{q}_{k}\right) \\
& \mathbf{d}_{k}=\mathbf{h}_{k}\left(\mathbf{x}_{k}, \boldsymbol{\epsilon}_{k}\right)
\end{aligned}
$$

where $\mathbf{q}_{k}$ and $\boldsymbol{\epsilon}_{k}$ are independent zero-mean random vectors.

The EnKF has the following algorithm: ${ }^{1}$

1. Create an ensemble $\left\{\mathbf{x}_{0, i}^{f}\right\}$ of size $N$ with $i=1, \ldots, N$, using the prior PDF of $\mathbf{x}_{0}$.

2. For each subsequent step, recursively, obtain perturbed measurements and estimated measurement error covariance matrix:

$$
\begin{aligned}
\mathbf{d}_{k, i} & =\mathbf{d}_{k}+\boldsymbol{\epsilon}_{k, i} \\
\boldsymbol{\Gamma}_{k} & =\frac{1}{N-1} \sum_{j=1}^{N} \boldsymbol{\epsilon}_{k, j} \boldsymbol{\epsilon}_{k, j}^{T}
\end{aligned}
$$

3. Analysis step:

$$
\begin{aligned}
\mathbf{C}_{k} & =\frac{\partial \mathbf{h}_{k}\left(\mathbf{x}_{k}, \boldsymbol{\epsilon}_{k}\right)}{\partial \mathbf{x}_{k}}, \\
\mathbf{D}_{k} & =\frac{\partial \mathbf{h}_{k}\left(\mathbf{x}_{k}, \boldsymbol{\epsilon}_{k}\right)}{\partial \boldsymbol{\epsilon}_{k}}, \\
\mathbf{K}_{k} & =\mathbf{P}_{k}^{f} \mathbf{C}_{k}^{T}\left[\mathbf{D}_{k} \boldsymbol{\Gamma}_{k} \mathbf{D}_{k}^{T}+\mathbf{C}_{k} \mathbf{P}_{k}^{f} \mathbf{C}_{k}^{T}\right]^{-1}, \\
\mathbf{x}_{k, i}^{a} & =\mathbf{x}_{k, i}^{f}+\mathbf{K}_{k}\left(\mathbf{d}_{k, i}-\mathbf{h}_{k}\left(\mathbf{x}_{k, i}^{f}, \boldsymbol{\epsilon}_{k, i}\right)\right), \\
\overline{\mathbf{x}}_{k}^{a} & \approx \frac{1}{N} \sum_{j=1}^{N} \mathbf{x}_{k, j}^{a}, \\
\mathbf{P}_{k}^{a} & =\left[\mathbf{I}-\mathbf{K}_{k} \mathbf{C}_{k}\right] \mathbf{P}_{k}^{f} .
\end{aligned}
$$

4. Forecast step:

$$
\begin{aligned}
\mathbf{x}_{k+1, i}^{f} & =\mathbf{\Psi}_{k}\left(\mathbf{x}_{k, i}^{a}, \mathbf{f}_{k}, \mathbf{q}_{k, i}\right), \\
\overline{\mathbf{x}}_{k+1}^{f} & \approx \frac{1}{N} \sum_{j=1}^{N} \mathbf{x}_{k+1, j}^{f}, \\
\mathbf{P}_{k+1}^{f} & \approx \frac{1}{N-1} \sum_{j=1}^{N}\left(\mathbf{x}_{k+1, j}^{f}-\overline{\mathbf{x}}_{k+1}^{f}\right)\left(\mathbf{x}_{k+1, j}^{f}-\overline{\mathbf{x}}_{k+1}^{f}\right)^{T} .
\end{aligned}
$$

In the above equations, the superscript $a$ denotes analysis and $f$ denotes forecast. In dealing with the nonlinear measurement operator, we have shown the traditional approach of linearizing the nonlinear measurement operator. For the case of additive measurement noise, the linearization can be avoided by augmenting the state vector with $\mathbf{h}_{k}\left(\mathbf{x}_{k}\right)$ as described by Evensen. ${ }^{15}$ For the numerical investigation conducted here, the measurement operator is linear and thus this linearisation step does not arise. 


\section{The Particle Filter}

Consider the model and measurement equations as described by Eqs. 1-2. The state and the measurement matrices are defined ${ }^{7,10,14}$ as

$$
\begin{aligned}
& \mathbf{X}_{k}=\left\{\mathbf{x}_{0}, \mathbf{x}_{1}, \ldots, \mathbf{x}_{k}\right\} \\
& \mathbf{D}_{k}=\left\{\mathbf{d}_{0}, \mathbf{d}_{1}, \ldots, \mathbf{d}_{k}\right\}
\end{aligned}
$$

By Bayes' Theorem, $7,10,14$ we have

$$
p\left(\mathbf{X}_{k} \mid \mathbf{D}_{k}\right)=\frac{p\left(\mathbf{D}_{k} \mid \mathbf{X}_{k}\right) p\left(\mathbf{X}_{k}\right)}{\int p\left(\mathbf{D}_{k} \mid \mathbf{X}_{k}\right) p\left(\mathbf{X}_{k}\right) \mathrm{d} \mathbf{X}_{k}} .
$$

Thus,

$$
p\left(\mathbf{x}_{k} \mid \mathbf{D}_{k}\right)=\frac{\int p\left(\mathbf{D}_{k} \mid \mathbf{X}_{k}\right) p\left(\mathbf{X}_{k}\right) \mathrm{d} \mathbf{X}_{k-1}}{\int p\left(\mathbf{D}_{k} \mid \mathbf{X}_{k}\right) p\left(\mathbf{X}_{k}\right) \mathrm{d} \mathbf{X}_{k}}
$$

One obtains the mean of the state vector by

$$
\overline{\mathbf{x}}_{k}=\frac{\int \mathbf{x}_{k} p\left(\mathbf{D}_{k} \mid \mathbf{X}_{k}\right) p\left(\mathbf{X}_{k}\right) \mathrm{d} \mathbf{X}_{k}}{\int p\left(\mathbf{D}_{k} \mid \mathbf{X}_{k}\right) p\left(\mathbf{X}_{k}\right) \mathrm{d} \mathbf{X}_{k}} .
$$

PF has the following algorithm:

1. Create an ensemble $\left\{\mathbf{x}_{0, i}^{f}\right\}$ of size $N$ with $i=1, \ldots, N$, using the prior PDF of $\mathbf{x}_{0}$.

2. Analysis step:

$$
\begin{aligned}
& \mathbf{x}_{k, i}^{a}=\mathbf{x}_{k, i}^{f}, \\
& w_{k, i}=\frac{p\left(\mathbf{d}_{k} \mid \mathbf{x}_{k, i}^{f}\right) w_{k-1, i}}{\sum_{j=1}^{N} p\left(\mathbf{d}_{k} \mid \mathbf{x}_{k, j}^{f}\right) w_{k-1, j}}, \\
& \overline{\mathbf{x}}_{k}^{a} \approx \sum_{i=1}^{N} w_{k, i} \mathbf{x}_{k, i}^{a}, \\
& \mathbf{P}_{k}^{a} \approx \sum_{j=1}^{N} w_{k, i}\left(\mathbf{x}_{k, j}^{a}-\overline{\mathbf{x}}_{k}^{a}\right)\left(\mathbf{x}_{k, j}^{a}-\overline{\mathbf{x}}_{k}^{a}\right)^{T} .
\end{aligned}
$$

3. Forecast step:

$$
\begin{aligned}
\mathbf{x}_{k+1, i}^{f} & =\mathbf{\Psi}_{k}\left(\mathbf{x}_{k, i}^{a}, \mathbf{f}_{k}, \mathbf{q}_{k, i}\right), \\
\overline{\mathbf{x}}_{k+1}^{f} & \approx \sum_{j=1}^{N} w_{k, i} \mathbf{x}_{k+1, j}^{f}, \\
\mathbf{P}_{k+1}^{f} & \approx \sum_{j=1}^{N} w_{k, i}\left(\mathbf{x}_{k+1, j}^{f}-\overline{\mathbf{x}}_{k+1}^{f}\right)\left(\mathbf{x}_{k+1, j}^{f}-\overline{\mathbf{x}}_{k+1}^{f}\right)^{T} .
\end{aligned}
$$

Eq. (19) implies the need for a choice for the values $w_{0, i}, i=1, \ldots, N$. One can start with $w_{0, i}=1 / N$. 


\section{A. Resampling}

In most practical applications of the particle filter, after a certain number of recursive steps, all but one particle (sample) will have negligible weights $w_{k, i}$. This is known as the degeneracy phenomenon. ${ }^{7}$ As a result of degeneracy, a large computational effort is wasted in updating particles which make little contribution to the state vector estimate. A suitable measure of degeneracy is the effective sample size given by

$$
N_{e f f}=\frac{1}{\sum_{i=1}^{N}\left(w_{k, i}\right)^{2}} .
$$

Degeneracy can be detected when when $N_{\text {eff }}$ falls below a threshold $N_{t h r}$. In that case, we perform resampling to reduce the degree of degeneracy:

1. Draw $N$ particles from the current particle set with probabilities equal to their weights $w_{k, i}$, replacing the current particle set with the new one.

2. Set $w_{k, i}=1 / N$ for $i=1, \ldots, N$.

\section{Application to Non-linear Dynamical Systems}

For numerical illustration, we consider a Duffing oscillator undergoing Limit Cycle Oscillation (LCO). In this section, we investigate the performance of EKF, EnKF and PF in tracking LCO. In particular, we examine the influence of measurement and model noise and sparsity of observational data on the tracking capabilities of EKF, EnKF and PF.

\section{A. Duffing oscillator model}

The equation of motion of a Duffing oscillator ${ }^{11}$ subjected to combined deterministic and random inputs is described as:

$$
\ddot{u}(t)+c \dot{u}(t)+k_{1} u(t)+k_{3} u^{3}(t)=\mathcal{T} \cos (\omega t)+\sigma_{1} \xi_{1}(t) .
$$

Here $c$ is the damping coefficient, $k_{1}$ and $k_{3}$ are the linear and nonlinear stiffness coefficients, $u(t)$ is the displacement, $\mathcal{T}$ and $\omega$ are the amplitude and frequency of the sinusoidal input, $\xi_{1}(t)$ is a Gaussian white noise describing the random input and $\sigma_{1}$ denotes its strength.

Despite its mathematical simplicity, the Duffing system displays a wide range of dynamics from periodic to chaotic motion with a slight change in its model parameters. For instance, when a Duffing system exhibits a double-well potential function for its autonomous counterpart, qualitatively different LCOs emerge by slight variations in the amplitude of the harmonic input $\mathcal{T}$ for the forced system. These facts prompted the use of the Duffing oscillator as a paradigm model in this investigation.

Using nonlinear filtering techniques, we are primarily interested in estimating the stiffness parameters leading to noisy LCOs of the Duffing oscillator from a set of noisy observational data obtained at discrete times $t_{k}$ :

$$
d_{k}=u\left(t_{k}\right)+\epsilon_{k} .
$$

Before we discuss joint state and parameter estimation using nonlinear filters, let us rewrite Eq. (27) in the state-space form as:

$$
\begin{aligned}
& \dot{x}_{1}=x_{2} \\
& \dot{x}_{2}=-\left[c x_{2}+k_{1} x_{1}+k_{3} x_{1}^{3}\right]+\mathcal{T} \cos (\omega t)+\sigma_{1} \xi_{1}(t),
\end{aligned}
$$


where $x_{1}=u$ and $x_{2}=\dot{u}$. The unknown parameters to be estimated are the stiffness coefficients $k_{1}$ and $k_{3}$. We augment the state vector by appending the coefficients $k_{1}$ and $k_{3}$ as two new state variables $x_{3}=k_{1}$ and $x_{4}=k_{3}$. The new variables are assumed to evolve using the following model

$$
\begin{aligned}
& \dot{x}_{3}=\sigma_{2} \xi_{2}(t) \\
& \dot{x}_{4}=\sigma_{3} \xi_{3}(t) .
\end{aligned}
$$

The following Itô Stochastic Differential Equations (SDEs) represents the above equations:

$$
\begin{aligned}
& d x_{1}=x_{2} d t \\
& d x_{2}=-\left[c x_{2}+x_{3} x_{1}+x_{4} x_{1}^{3}-\mathcal{T} \cos (\omega t)\right] d t+\sigma_{1} \xi_{1}(t) d t \\
& d x_{3}=\sigma_{2} \xi_{2}(t) d t \\
& d x_{4}=\sigma_{3} \xi_{3}(t) d t
\end{aligned}
$$

where $\xi_{j}(t) d t=d W_{j}=W_{j}\left(t_{k+1}\right)-W_{j}\left(t_{k}\right)$ are Brownian path increments.

The discrete representation the above coupled Itô SDEs using the Euler-Maruyama stochastic integration scheme ${ }^{16}$ with time step $\Delta t$ provides the following discrete state-evolution equations:

$$
\begin{aligned}
& \left\{x_{1}\right\}_{k+1}=\left\{x_{1}\right\}_{k}+\Delta t\left\{x_{2}\right\}_{k} \\
& \left\{x_{2}\right\}_{k+1}=\left\{x_{2}\right\}_{k}-\Delta t\left[c\left\{x_{2}\right\}_{k}+\left\{x_{3}\right\}_{k}\left\{x_{1}\right\}_{k}+\left\{x_{4}\right\}_{k}\left\{x_{1}\right\}_{k}^{3}-\mathcal{T} \cos \left(\omega t_{k}\right)\right]+\sigma_{1} \sqrt{\Delta t} \varepsilon_{1, k} \\
& \left\{x_{3}\right\}_{k+1}=\left\{x_{3}\right\}_{k}+\sigma_{2} \sqrt{\Delta t} \varepsilon_{2, k} \\
& \left\{x_{4}\right\}_{k+1}=\left\{x_{4}\right\}_{k}+\sigma_{3} \sqrt{\Delta t} \varepsilon_{3, k}
\end{aligned}
$$

where $\varepsilon_{1, k}, \varepsilon_{2, k}$ and $\varepsilon_{3, k}$ are independent standard Gaussian random variables. The measurement equation remains the same as in Equation (28).

The purpose of introducing the perturbation terms whose amplitudes are $\sigma_{2}$ and $\sigma_{3}$ is to inflate the variance of the parameter estimates and thus avoid filter divergence. Several methods are reported in the literature for inflating the estimates: ${ }^{17}$ (1) Additive inflation in which noise is added to the estimates; (2) Multiplicative inflation where the estimate covariance matrix is multiplied by a constant factor, usually greater than one; (3) Model-specific inflation where only a subset of the model parameters are perturbed. In this investigation, we employ model-specific additive inflation. From extensive numerical experiments, the values $\sigma_{2}=0.03$ and $\sigma_{3}=0.03$ lead to rapid convergence of the filter estimates. Setting these values too large lead to divergence of the estimates, whereas the convergence of the estimates is slower for smaller values of these parameters.

\section{B. LCO of the Duffing oscillator}

Next we investigate the LCO of the Duffing system. In particular, we examine one subharmonic $\mathrm{LCO}$ as described later in this section. The following numerical values of the system parameters are considered: $c=0.3, k_{1}=-1, k_{3}=1, \omega=1.25$ and $\Delta t=5 \times 10^{-4}$. For this parameter set, the autonomous system has three fixed points. The fixed point at $u=0$ is unstable, while the two fixed points at \pm 1 are stable. Fig. 1 displays the steady-state displacement $u$ and its phasespace diagram under purely deterministic loading $(\sigma=0)$ for $\mathcal{T}=0.3$. The two trajectories in Fig. 1 represent the steady state LCOs about the stable fixed points, starting with different 
initial conditions. In the absence of modelling noise $(\sigma=0)$, when $\mathcal{T}=0.3$, the system exhibits a period-two subharmonic LCO with period $4 \pi / \omega$.

Fig. 2 presents sample trajectories and the associated phase-space diagram of the noisy LCO perturbed by weak random noise $(\sigma=0.01)$ with $\mathcal{T}=0.3$. Note that the transient response associated with the initial conditions is not shown. Under such mild random perturbation, the periodic response of LCO is gently disturbed as evident in the banded structure in the phasespace diagrams. In other words, the regularity of the LCO largely remains intact as observed in the left panels of Fig. 2.
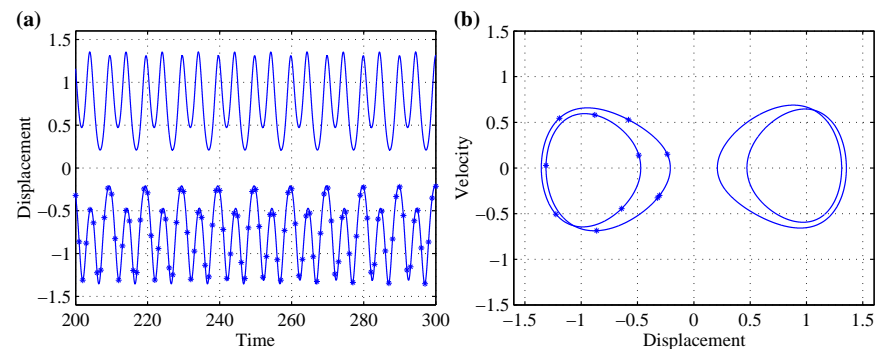

Figure 1. Response of the Duffing oscillator under purely deterministic input with $\mathcal{T}=0.3, \sigma=0$ : (a) two steady-state trajectories, (b) the associated phase-space diagrams
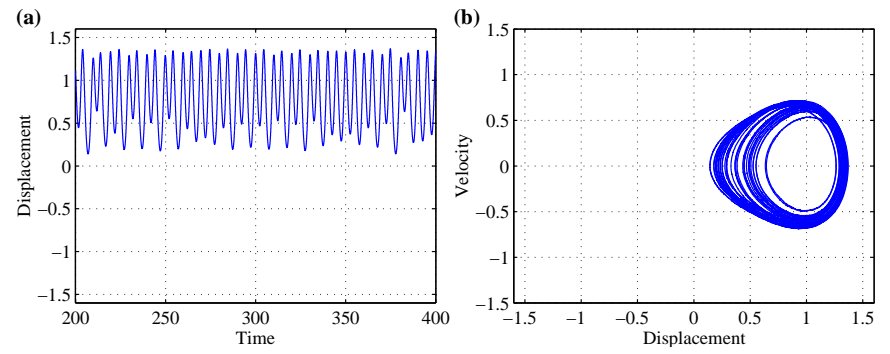

Figure 2. Response of the Duffing oscillator under combined deterministic and random input with $\mathcal{T}=0.3, \sigma=0.01$ : (a) steady-state trajectory, (b) the associated phase-space diagram

\section{Transition PDFs of LCO}

The transition PDF of the system driven purely by random noise $(\mathcal{T}=0$ and $\sigma=0.01)$ is shown in Fig. 3 with initial conditions $x_{1} \sim \mathrm{U}[-2,2]$ and $x_{2}=0$. The transition PDF is obtained using $3 \times 10^{6}$ Monte-Carlo samples running in parallel on a shared-memory multiprocessor machine (SGI Altix350) using message-passing interface (MPI). ${ }^{18}$ To expedite the statistical convergence with fewer samples, Latin Hypercube Sampling (LHS) was employed as an efficient sampling technique. The initial uniform PDF of $x_{1}$ converges to a bimodal (strongly non-Gaussian) stationary distribution having dominant peaks at the stable fixed points $u= \pm 1$.

Next we turn our attention to the forced system. The transition PDF of the displacement $x_{1}$ is plotted in Fig. 4 under combined deterministic and random excitations $(\sigma=0.01)$. The transition PDFs are generated using $3 \times 10^{6}$ random samples using LHS. Initial conditions are $x_{1} \sim \mathrm{U}[-2,2]$ and $x_{2}=0$. The strongly non-Gaussian trends in the transition PDFs are clearly evident in Fig. 4. In Fig. 4, the probability mass is centred around the two stable fixed 
points $u= \pm 1$. This is due to the fact that the oscillation is predominantly confined in the neighborhood of potential wells around $u= \pm 1$.

Nonlinear filtering techniques estimate the conditional PDFs of the system given noisy observational data. The effective performance of these filters relies heavily on the characteristics of the conditional PDFs. For example, strong non-Gaussian features in the conditional PDFs diminishes the efficacy of EKF. On the contrary, PF is robust to non-Gaussian trends. It is therefore instructive to focus on the nature of the conditional PDF to judiciously apply nonlinear filters. Some examples of the conditional PDF are presented in the bottom panels of Fig. 5 using two measurements $d_{k} \pm 0.5$ corrupted by Gaussian noise for $\mathcal{T}=0.3$. The variance of the zero-mean Gaussian measurement noise is taken to be 0.1. The initial and transition PDF are shown in the top panel of Fig. 5. Strong non-Gaussian features in the conditional PDF are clearly evident which point out the need to use nonlinear filtering techniques such as EnKF and PF.
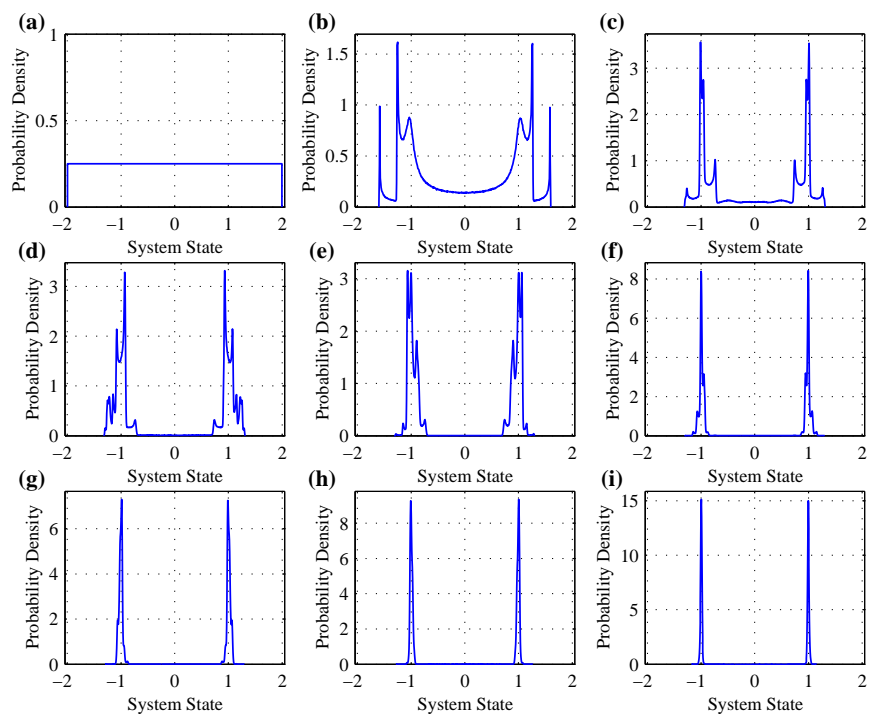

Figure 3. Transition PDF of the system under purely random input with $\sigma=0.01$ and $\mathcal{T}=0$ : (a) Initial uniform $\mathrm{PDF}$, (b) PDF at $t=3$ time units, (c) PDF at $t=6$ time units, (d) PDF at $t=9$ time units, (e) PDF at $t=12$ time units, (f) PDF at $t=15$ time units, (g) PDF at $t=18$ time units, (h) PDF at $t=21$ time units, (i) PDF at $t=24$ time units

\section{Joint State and Parameter Estimation}

Next we conduct extensive numerical experiments related to joint parameter and state estimation of LCO using nonlinear filters. In Fig. 6, the top panel shows the displacement of the oscillator and the associated observational data $d_{k}$ contaminated by Gaussian noise $\epsilon_{k} \sim \mathcal{N}\left(0,6.2 \times 10^{-3}\right)$ for the case of $\mathcal{T}=0.3$. The standard deviation of the measurement noise is taken to be $10 \%$ of the root-mean-square (RMS) value of the true displacement. The modelling error has an amplitude of $\sigma=0.02$. The observational data is acquired and analyzed at intervals of 1 time unit. For EnKF and PF, an ensemble size of $N=1000$ is used. To further minimize sampling errors, LHS ${ }^{19,20}$ is employed as an efficient sampling scheme by both EnKF and PF.

The result of the joint state and parameter estimation are shown in Figures 7-8. Initial conditions are $x_{1} \sim \mathcal{N}(0,0.01), x_{2} \sim \mathcal{N}(0,0.01), k_{1}=x_{3} \sim \mathcal{N}(-0.75,0.01)$ and $k_{3}=$ 

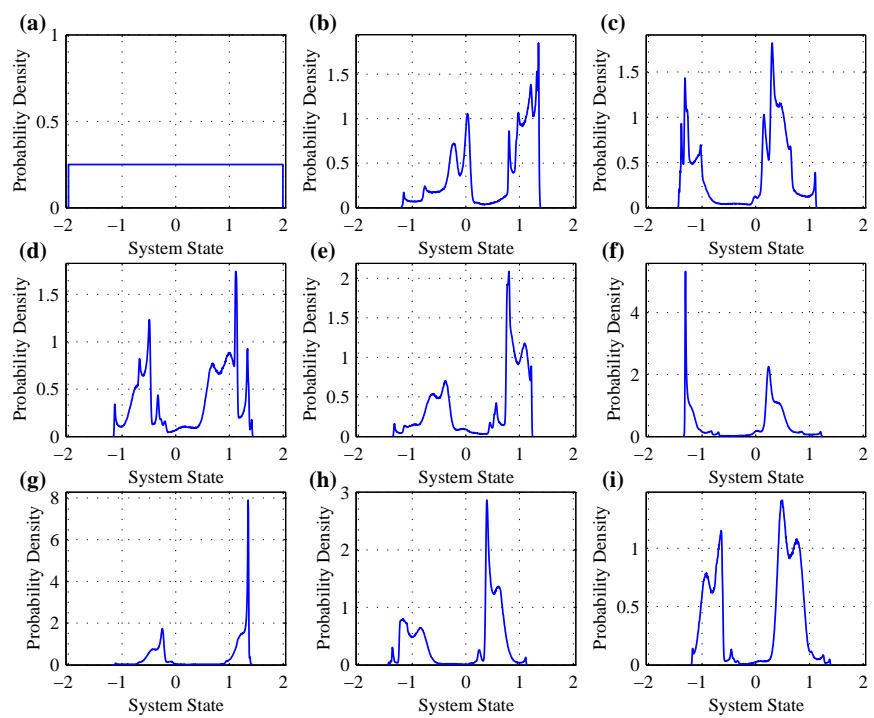

Figure 4. Transition PDF of the system under combined deterministic and random input with $\sigma=0.01$ and $\mathcal{T}=0.3$ : (a) Initial uniform PDF, (b) PDF at $t=12$ time units, (c) PDF at $t=24$ time units, (d) PDF at $t=36$ time units, (e) PDF at $t=48$ time units, (f) PDF at $t=60$ time units, (g) PDF at $t=72$ time units, (h) PDF at $t=84$ time units, (i) $\mathrm{PDF}$ at $t=96$ time units
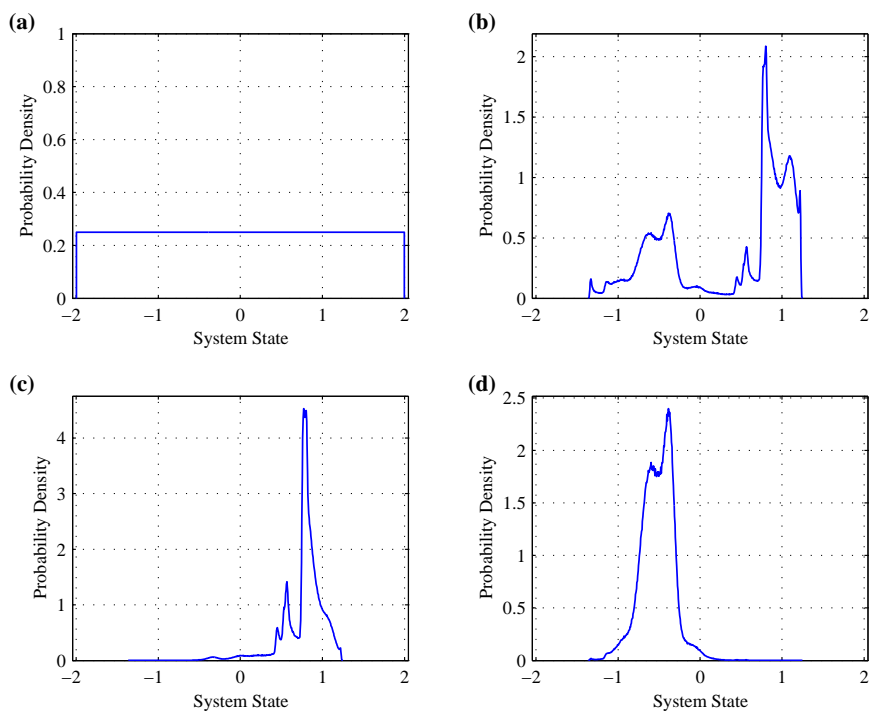

Figure 5. Conditional PDF of the system with $\sigma=0.01$ and $\mathcal{T}=0.3$ : (a) Initial uniform PDF, (b) prior PDF at $t=48$ time units, (c) conditional PDF given measurement $d_{k}=0.5$, (d) conditional PDF given measurement $d_{k}=-0.5$

$x_{4} \sim \mathcal{N}(0.75,0.01)$. The choice of initial conditions is another factor that influences the convergence of the filters. This set of initial conditions is chosen to illustrate the ability of the filtering algorithms to successfully track the system even with inaccurate initial estimates of the parameters. If the initial conditions are grossly inaccurate, the filters may diverge in which case one has to rerun the filters with different initial conditions. Normally, the prior knowledge, whenever available, should influence the choice of initial conditions.

The estimates of displacement of the oscillator using EKF (second panel from the top), EnKF 
(third panel from the top) and PF (fourth panel from the top) are plotted in Figure 6. The moving average of the normalized RMS errors of the estimates are plotted in the bottom-most panel of Fig. 6. The error is normalized by the variance of the true displacement and the average is taken over the last 50 time units. The threshold effective ensemble size for PF for resampling purposes is chosen to be $75 \%$ of the ensemble size (i.e. $N_{t h r}=0.75 N$ ). From the experience gained by the authors through numerical investigations, this value for the threshold ensemble size turned out to be adequate for effective resampling. All three filters give satisfactory results. The estimates for the stiffness coefficients $k_{1}$ and $k_{3}$ are plotted in Figures 7-8, respectively. EKF estimates are plotted in the first panel from the top, EnKF estimates in the second panel from the top, and PF estimates are plotted in the third panel from the top. Again, all three filters give similar estimates. While comparing the standard deviation of the error in the estimates, it is clear that PF leads to smaller error standard deviation.

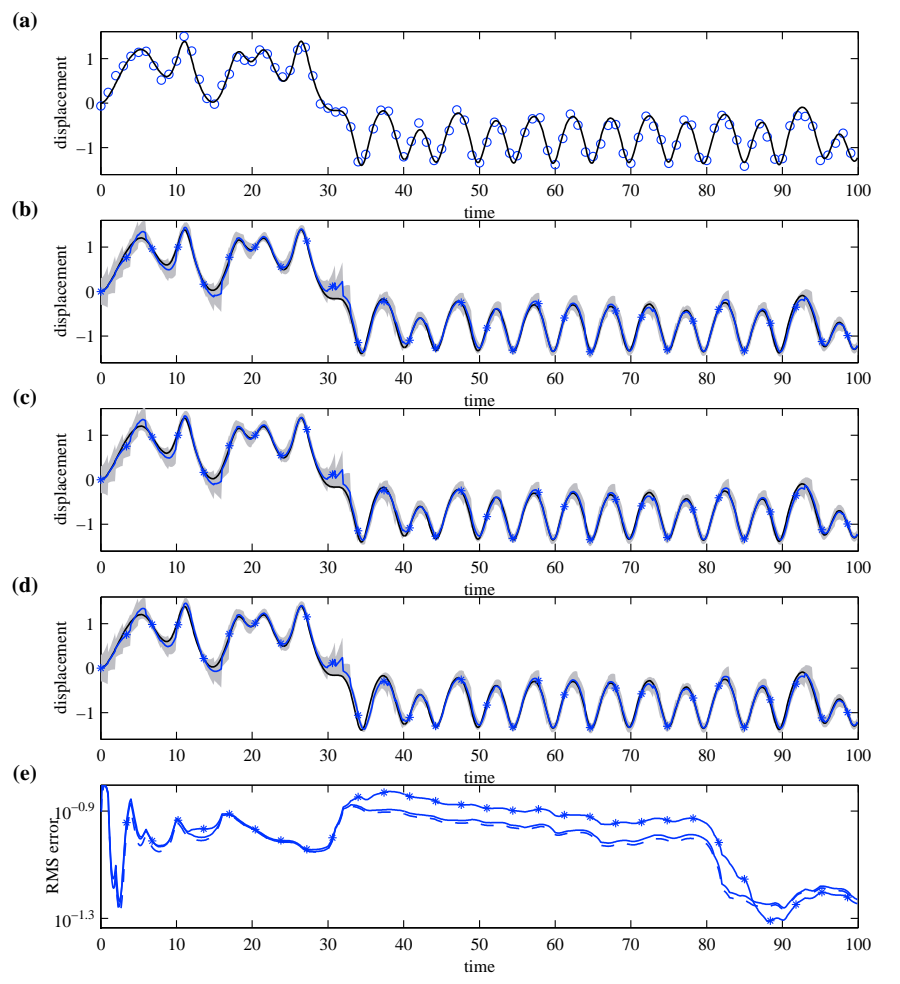

Figure 6. True, measured and estimated displacement of the Duffing oscillator: (a) true (solid line) and measured (circles) displacement, (b) EKF estimate (blue line with stars) \pm 3 error standard deviations (light blue area), (c) EnKF estimate (blue line with stars) \pm 3 error standard deviations (light blue area) with $N=1000$, (d) PF estimate (blue line with stars) \pm 3 error standard deviations (light blue area) with $N=1000$, (e) time-averaged normalized RMS estimate errors of EKF (dashed line), EnKF (solid line) and PF (solid line with stars)

\section{Effect of ensemble size}

To demonstrate the effect of ensemble size for EnKF and PF on the state and parameter estimates, we consider the observational data acquired at every 1 time unit contaminated by Gaussian noise $\epsilon_{k} \sim \mathcal{N}\left(0,6.2 \times 10^{-3}\right)$, same as in the previous experiment, but with a smaller ensemble size of $N=10$ for EnKF and PF, in contrast to $N=1000$ used in the previous experiment. The estimates of displacement of the oscillator using EKF (second panel from the top), EnKF (third panel from the top) and PF (fourth panel from the top) are plotted in 

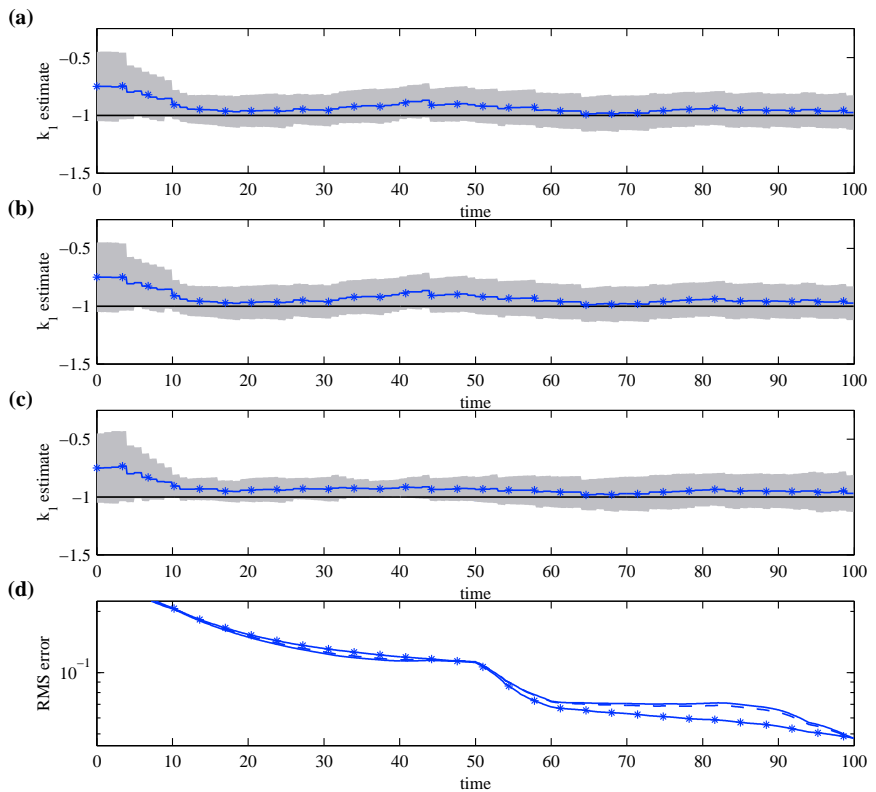

Figure 7. $k_{1}$ parameter estimates: (a) EKF estimate (blue line with stars) \pm 3 error standard deviations (light blue area), (b) EnKF estimate (blue line with stars) \pm 3 error standard deviations (light blue area) with $N=1000$, (c) $\mathrm{PF}$ estimate (blue line with stars) \pm 3 error standard deviations (light blue area) with $N=1000$, (e) time-averaged normalized RMS estimate errors of EKF (dashed line), EnKF (solid line) and PF (solid line with stars)

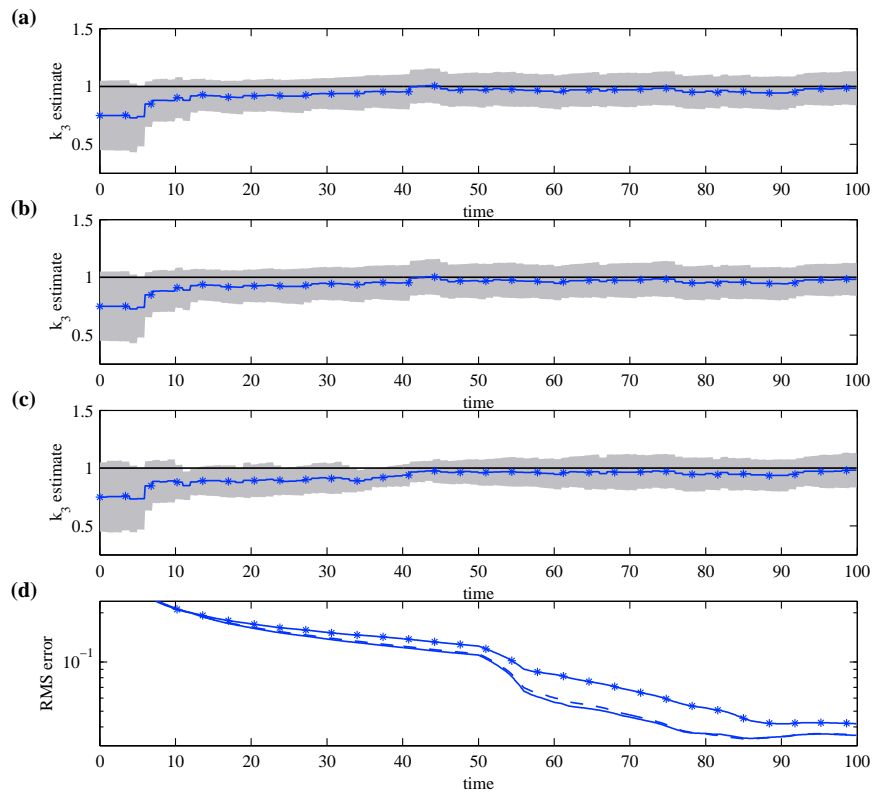

Figure 8. $k_{3}$ parameter estimates: (a) EKF estimate (blue line with stars) \pm 3 error standard deviations (light blue area), (b) EnKF estimate (blue line with stars) \pm 3 error standard deviations (light blue area) with $N=1000,($ c) $\mathrm{PF}$ estimate (blue line with stars) \pm 3 error standard deviations (light blue area) with $N=1000$, (e) time-averaged normalized RMS estimate errors of EKF (dashed line), EnKF (solid line) and PF (solid line with stars)

Figure 9. The moving average of the normalized RMS errors of the estimates are plotted in the bottom-most panel of Fig. 9. The estimates for the stiffness coefficients $k_{1}$ and $k_{3}$ are plotted 
in Figures 10-11, respectively. EKF estimates are plotted in the first panel from the top, EnKF estimates in the second panel from the top, and PF estimates are plotted in the third panel from the top. Comparing the estimates obtained in this experiment with the ones obtained from the previous experiment, it is obvious that decreasing the ensemble size from $N=1000$ samples to $N=50$ significantly degrades the performance of PF. PF also results in smaller error variance estimates with a smaller ensemble size. In contrast, EnKF provides similar estimates using a smaller ensemble size of $N=50$ when compared to $N=1000$.

(a)

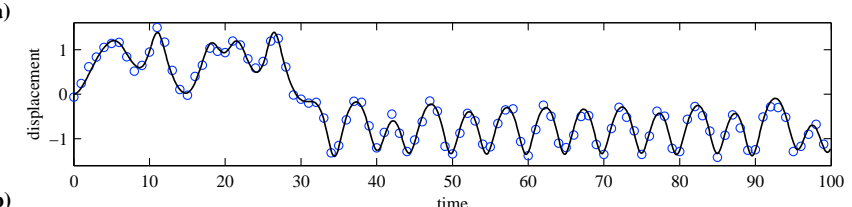

(b)
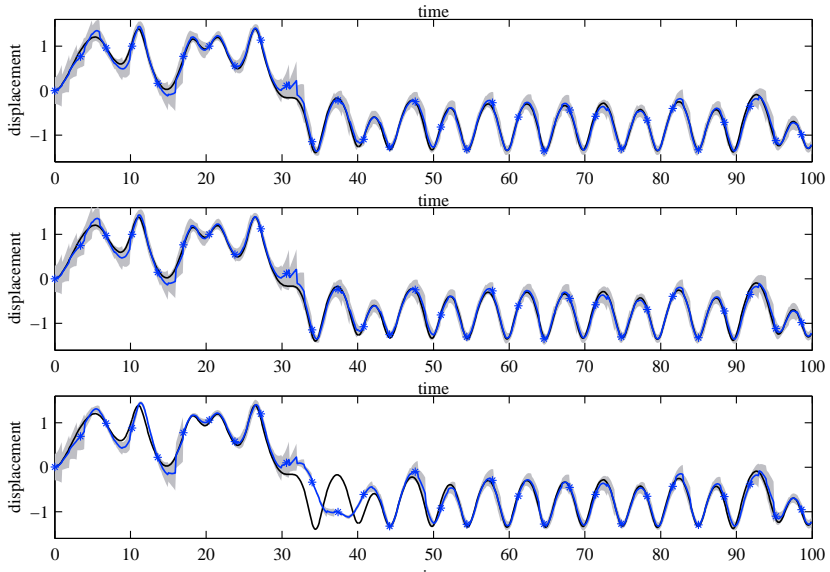

(e)

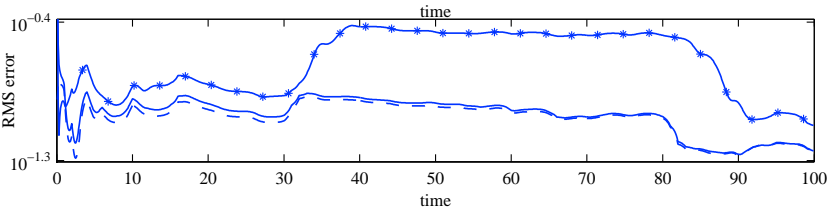

Figure 9. True, measured and estimated displacement of the Duffing oscillator using a smaller ensemble for EnKF and PF: (a) true (solid line) and measured (circles) displacement, (b) EKF estimate (blue line with stars) \pm 3 error standard deviations (light blue area), (c) EnKF estimate (blue line with stars) \pm 3 error standard deviations (light blue area) with $N=50$, (d) PF estimate (blue line with stars) \pm 3 error standard deviations (light blue area) with $N=50$, (e) time-averaged normalized RMS estimate errors of EKF (dashed line), EnKF (solid line) and PF (solid line with stars)

\section{Effect of measurement noise}

To demonstrate the effect of measurement noise on the state estimates, we consider the observational data acquired at every 1 time unit, same as in the previous experiment, but with stronger measurement noise, in contrast to the previous experiment. Fig. 12 shows the displacement of the oscillator for $\mathcal{T}=0.3$ and the measured displacement $d_{k}$ contaminated by Gaussian noise $\epsilon_{k} \sim \mathcal{N}\left(0,6.2 \times 10^{-1}\right)$. The variance of the measurement noise is taken to be equal to the mean-square value of the true displacement.

The state estimation results using EKF, EnKF and PF are shown in the second, third and fourth panels from the top in Fig. 12. In relation to the results from the first experiment shown in Fig. 6, it is evident that an increase in the strength of measurement noise deteriorates the accuracy of the estimates for all filters, although PF provides more accurate results than EnKF. 

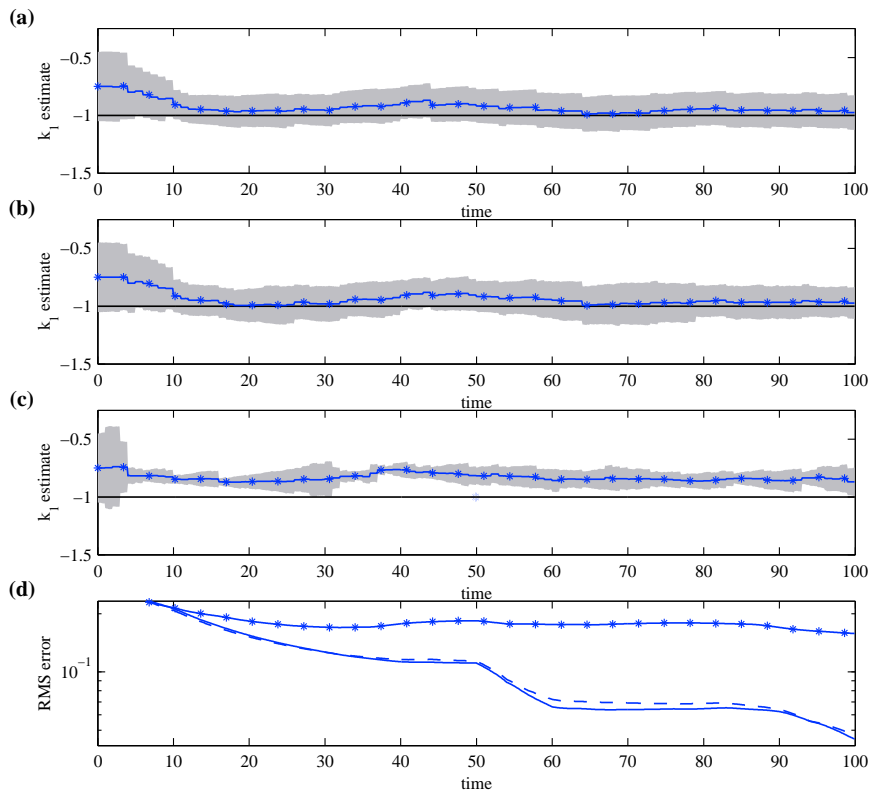

Figure 10. $k_{1}$ parameter estimates using a smaller ensemble for EnKF and PF: (a) EKF estimate (blue line with stars) \pm 3 error standard deviations (light blue area), (b) EnKF estimate (blue line with stars) \pm 3 error standard deviations (light blue area) with $N=50$, (c) PF estimate (blue line with stars) \pm 3 error standard deviations (light blue area) with $N=50$, (e) time-averaged normalized RMS estimate errors of EKF (dashed line), EnKF (solid line) and PF (solid line with stars)
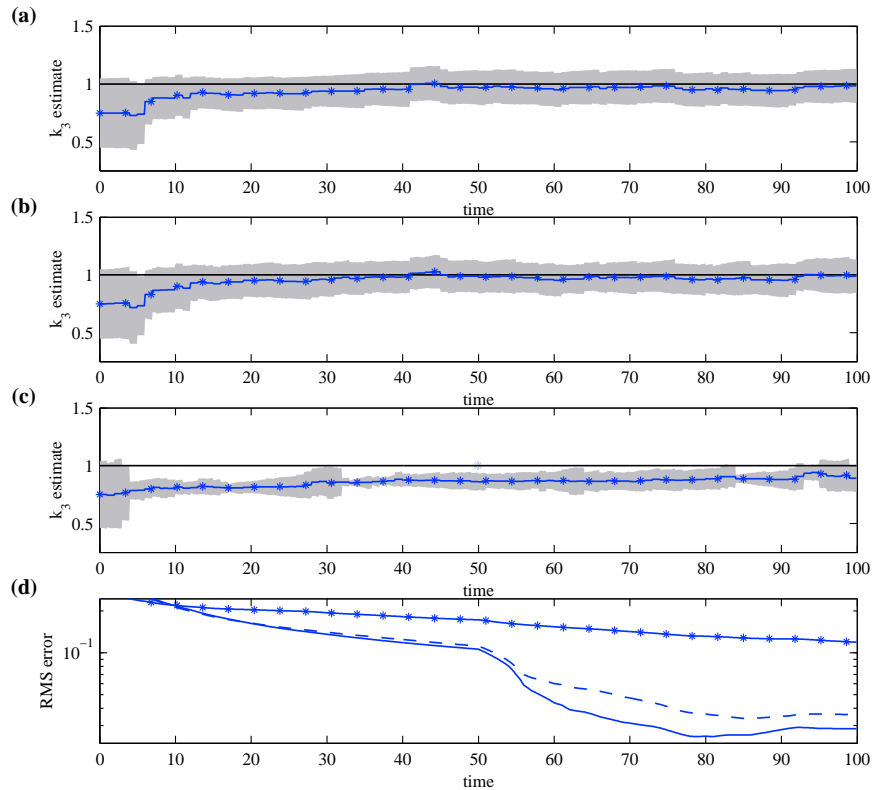

Figure 11. $k_{3}$ parameter estimates using a smaller ensemble for EnKF and PF: (a) EKF estimate (blue line with stars) \pm 3 error standard deviations (light blue area), (b) EnKF estimate (blue line with stars) \pm 3 error standard deviations (light blue area) with $N=50$, (c) PF estimate (blue line with stars) \pm 3 error standard deviations (light blue area) with $N=50$, (e) time-averaged normalized RMS estimate errors of EKF (dashed line), EnKF (solid line) and PF (solid line with stars) 
The estimates of EnKF are moderately accurate. The performance of EKF is the worst. It is believed that the superior performance of $\mathrm{PF}$ in this experiment is due to stronger non-Gaussian features in the conditional PDF in comparison to the previous case. This maybe inferred, for instance, looking at the conditional PDFs plotted in Fig. 5 where the increase in measurement noise more effectively retains the non-Gaussian features in the posterior PDF. In contrast to the previous case, the error standard deviation of the estimates is greater for all three filters. The increase in error standard deviation of the estimates is due to an increase in the variance of the measurement noise. PF estimates display smaller error covariances when compared to those of EKF and EnKF.

The estimates for the stiffness coefficients $k_{1}$ and $k_{3}$ are plotted in Figures 13-14, respectively. EKF estimates are plotted in the first panel from the top, EnKF estimates in the second panel from the top, and PF estimates are plotted in the third panel from the top. Comparing the estimates obtained in this experiment to those from the first experiment, an increase in measurement noise intensity leads to divergence for EKF and poor convergence for EnKF. PF gives the most accurate parameter estimates in this case.

(a)
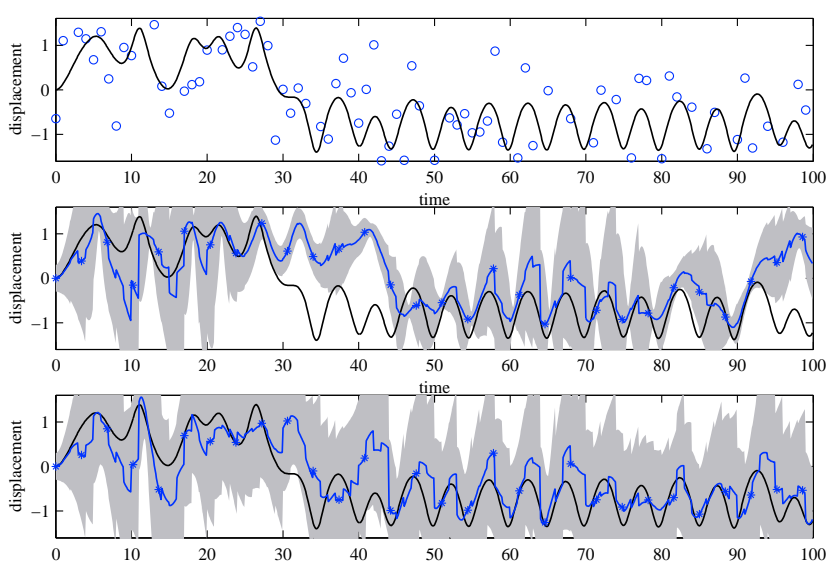

(d)
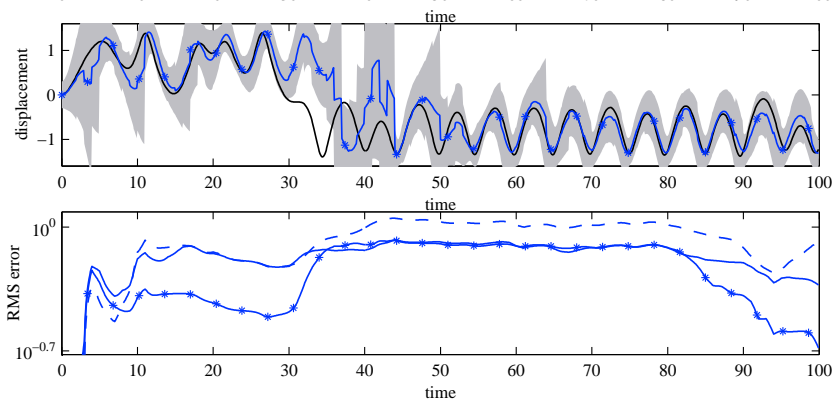

Figure 12. True, measured and estimated displacement of the Duffing oscillator with stronger measurement noise: (a) true (solid line) and measured (circles) displacement, (b) EKF estimate (blue line with stars) \pm 3 error standard deviations (light blue area), (c) EnKF estimate (blue line with stars) \pm 3 error standard deviations (light blue area) with $N=1000$, (d) PF estimate (blue line with stars) \pm 3 error standard deviations (light blue area) with $N=1000$, (e) time-averaged normalized RMS estimate errors of EKF (dashed line), EnKF (solid line) and PF (solid line with stars)

\section{Effect of observational data sparsity}

In this subsection, we consider the case of sparse observational data. In particular, we consider observations obtained at time intervals of 4 time units, instead of 1 time unit used in the 

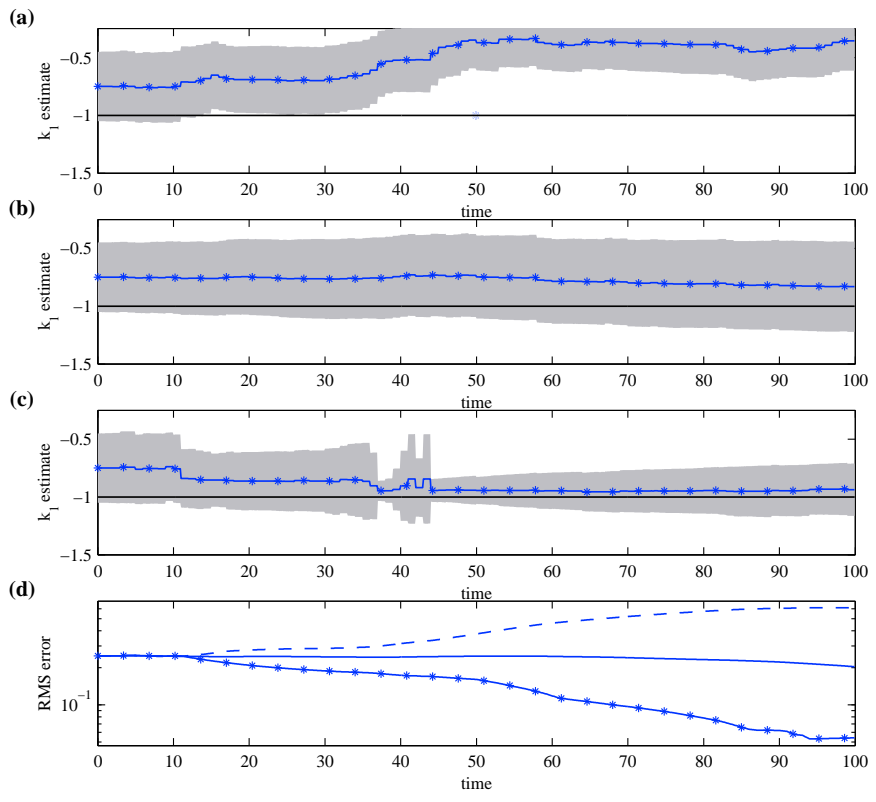

Figure 13. $k_{1}$ parameter estimates with stronger measurement noise: (a) EKF estimate (blue line with stars) \pm 3 error standard deviations (light blue area), (b) EnKF estimate (blue line with stars) \pm 3 error standard deviations (light blue area) with $N=1000$, (c) PF estimate (blue line with stars) \pm 3 error standard deviations (light blue area) with $N=1000$, (e) time-averaged normalized RMS estimate errors of EKF (dashed line), EnKF (solid line) and PF (solid line with stars)
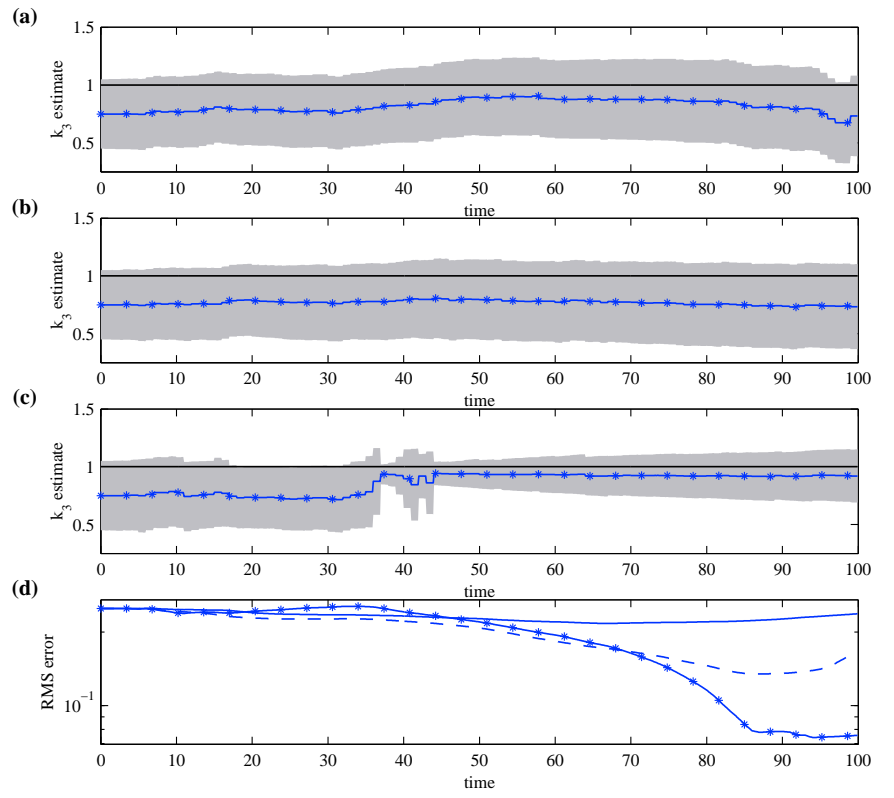

Figure 14. $k_{3}$ parameter estimates with stronger measurement noise: (a) EKF estimate (blue line with stars) \pm 3 error standard deviations (light blue area), (b) EnKF estimate (blue line with stars) \pm 3 error standard deviations (light blue area) with $N=1000$, (c) PF estimate (blue line with stars) \pm 3 error standard deviations (light blue area) with $N=1000$, (e) time-averaged normalized RMS estimate errors of EKF (dashed line), EnKF (solid line) and PF (solid line with stars) 
previous experiments. The standard deviation of measurement noise is $10 \%$ of that of the RMS value of the true displacement. The true and measured displacement are shown in the top most panel in Fig. 15. The state estimates of EKF, EnKF and PF are shown in subplots (b) through (d) in Fig. 15. Similar to the last experiment, EKF again leads to highly biased estimates, in this case due to an increase in data sparsity. PF provides slightly more accurate results than EnKF. The infrequent assimilation of data permits the PDF of the state variables to regain its non-Gaussian features over the measurement interval as observed in the transition PDF as in Fig. 4. Such non-Gaussian trends degrade the performance of EKF.

The estimates for the stiffness coefficients $k_{1}$ and $k_{3}$ are plotted in Figures 16-17, respectively. EKF estimates are plotted in the first panel from the top, EnKF estimates in the second panel from the top, and PF estimates are plotted in the third panel from the top. As in the previous experiment, EKF estimates diverge with an increase in data sparsity. EnKF PF provide similarly accurate parameter estimates.

(a)

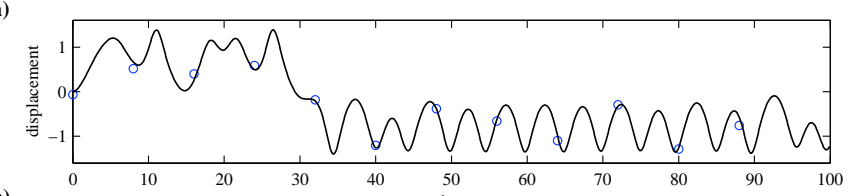

(b)
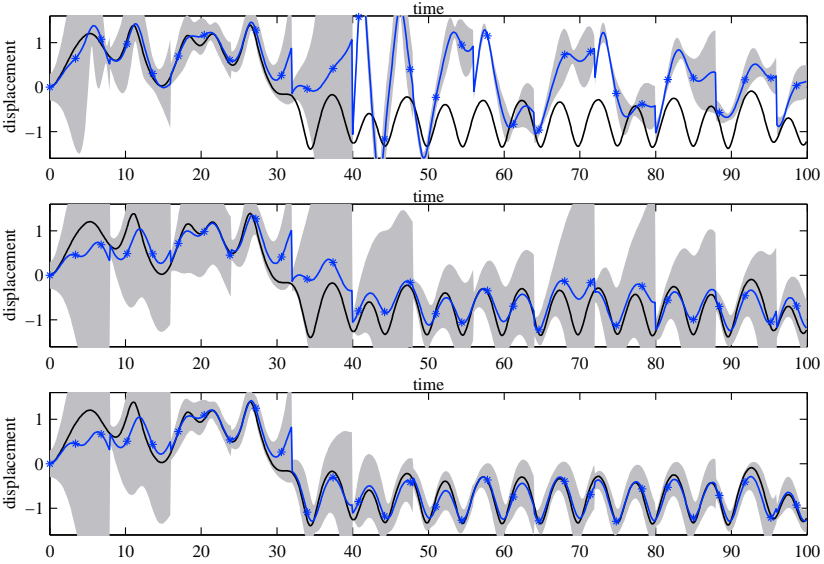

(e)

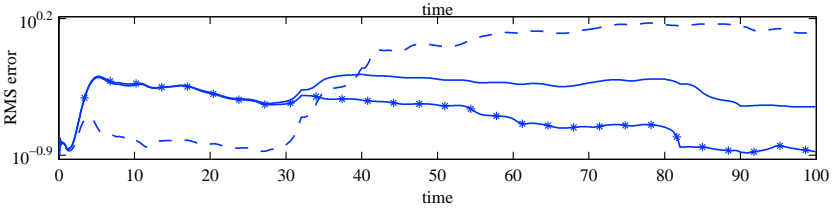

Figure 15. True, measured and estimated displacement of the Duffing oscillator with sparse observational data: (a) true (solid line) and measured (circles) displacement, (b) EKF estimate (blue line with stars) \pm 3 error standard deviations (light blue area), (c) EnKF estimate (blue line with stars) \pm 3 error standard deviations (light blue area) with $N=1000$, (d) PF estimate (blue line with stars) \pm 3 error standard deviations (light blue area) with $N=1000$, (e) time-averaged normalized RMS estimate errors of EKF (dashed line), EnKF (solid line) and PF (solid line with stars)

\section{Conclusion}

This paper investigates nonlinear filtering techniques, namely EKF, EnKF and PF, for the joint state and parameter estimation of noisy LCO. For the LCO of a Duffing system, extensive numerical experiments are conducted to bring out the superiority and limitations of these filters 

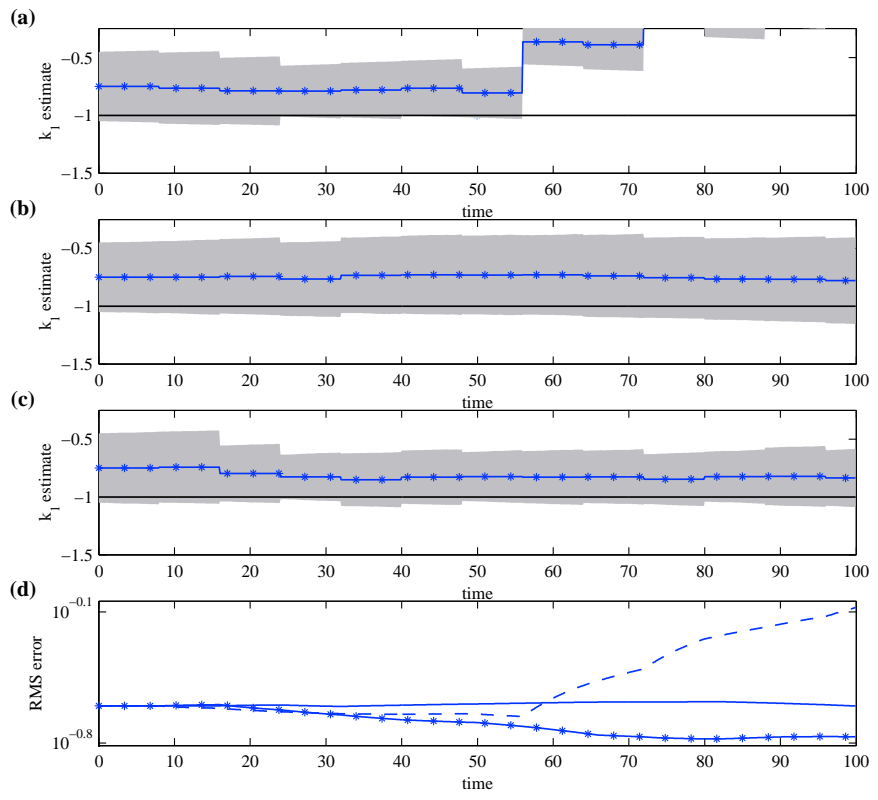

Figure 16. $k_{1}$ parameter estimates with sparse observational data: (a) EKF estimate (blue line with stars) \pm 3 error standard deviations (light blue area), (b) EnKF estimate (blue line with stars) \pm 3 error standard deviations (light blue area) with $N=1000$, (c) PF estimate (blue line with stars) \pm 3 error standard deviations (light blue area) with $N=1000$, (e) time-averaged normalized RMS estimate errors of EKF (dashed line), EnKF (solid line) and PF (solid line with stars)

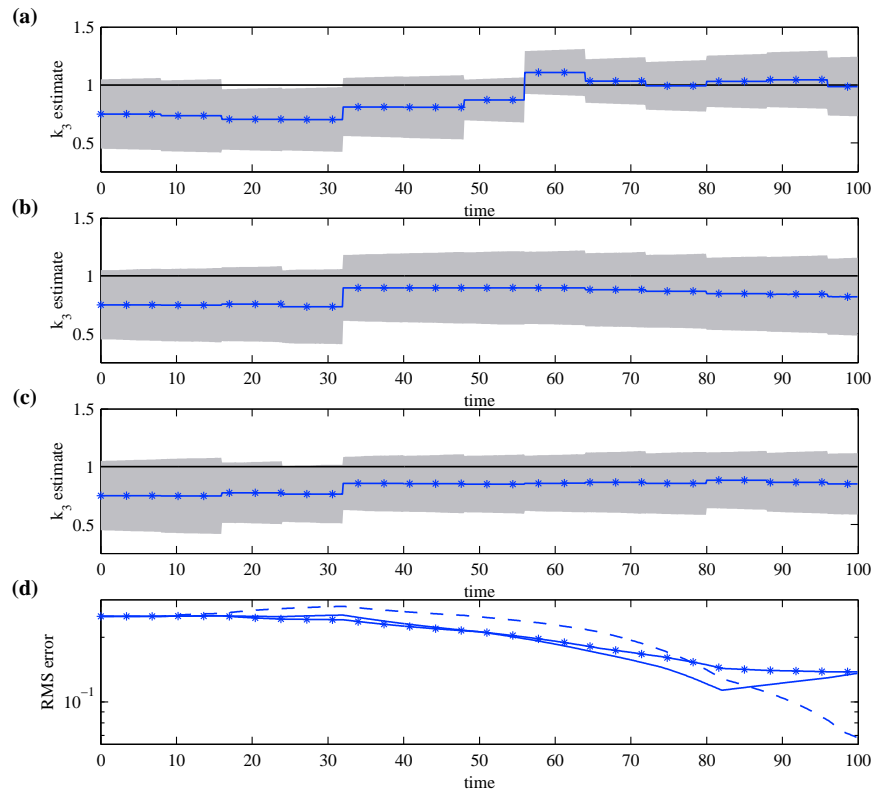

Figure 17. $k_{3}$ parameter estimates with sparse observational data: (a) EKF estimate (blue line with stars) \pm 3 error standard deviations (light blue area), (b) EnKF estimate (blue line with stars) \pm 3 error standard deviations (light blue area) with $N=1000$, (c) PF estimate (blue line with stars) \pm 3 error standard deviations (light blue area) with $N=1000$, (e) time-averaged normalized RMS estimate errors of EKF (dashed line), EnKF (solid line) and PF (solid line with stars) 
with respect to ensemble size as well as measurement errors and sparsity of observational data. The salient features emerging from this investigation are:

1. EKF provides similarly accurate state and parameter estimates in comparison to EnKF and $\mathrm{PF}$ in the case of relatively dense observational data and small measurement noise. The accuracy of EKF is due to the highly Gaussian nature of the state vector in the presence of dense observational data contaminated by relatively weak Gaussian noise.

2. The performance of PF turns out to be superior to EKF and EnKF when the measurement noise increases. EKF is most severely affected by an increase in measurement noise. The accuracy of the EnKF estimates is moderate (being superior to EKF but inferior to $\mathrm{PF}$ ). This can be explained from the fact that an increase in the strength of Gaussian measurement noise translates to a conditional pdf featuring stronger non-Gaussian traits. Being a fully non-Gaussian filter, PF provides the best estimates.

3. The increase in data sparsity has a detrimental effect on the state estimates of EKF. Increased data sparsity has little effect on the EnKF and PF estimates in the case examined.

\section{Acknowledgments}

The first author acknowledges the support of the National Sciences and Engineering Research Council of Canada through the award of a Canada Graduate Scholarship. The second author acknowledges the support of a Discovery Grant from National Sciences and Engineering Research Council of Canada and the Canada Research Chair Program. The third author acknowledges the support of the UK Engineering and Physical Sciences Research Council (EPSRC) through the award of an Advanced Research Fellowship and the Royal Society of London for the award of a visiting fellowship at Carleton University, Canada.

\section{References}

${ }^{1}$ Evensen, G., Data Assimilation: The Ensemble Kalman Filter, Springer, Berlin, 2006.

${ }^{2}$ Kaipio, J. and Somersalo, E., Statistical and Computational Inverse Problems, Springer, New York, 2005 .

${ }^{3}$ Bennett, A. F., Inverse Modeling of the Ocean and the Atmosphere, Cambridge University Press, Cambridge, U.K., 2002.

${ }^{4}$ Kalman, R. E., "A new approach to linear filtering and prediction problems," Journal of Basic Engineering, Vol. 82, 1960, pp. 35-45.

${ }^{5}$ Jazwinski, A. H., Stochastic Processes and Filtering Theory, Academic Press, San Diego, California, 1970.

${ }^{6}$ Evensen, G., "Using the extended Kalman filter with a multilayer quasi-geostrophic ocean model," Journal Of Geophysical Research - Oceans, Vol. 97, No. C11, 1992, pp. 17905-17924.

${ }^{7}$ Doucet, A., Godsill, S. J., and Andrieu, C., "On sequential Monte Carlo sampling methods for Bayesian filtering," Statistics and Computing, Vol. 10, No. 3, 2000, pp. 197-208.

${ }^{8}$ Ghanem, R. and Ferro, G., "Health monitoring for strongly non-linear systems using the ensemble Kalman filter," Structural Control and Health Monitoring, Vol. 13, No. 1, 2006, pp. 245-259.

${ }^{9}$ Miller, R. N., Carter, E. F., and Blue, S. T., "Data assimilation into nonlinear stochastic models," Tellus Series A - Dynamic Meteorology And Oceanography, Vol. 51, No. 2, 1999, pp. $167-194$.

${ }^{10}$ Manohar, C. S. and Roy, D., "Monte Carlo filters for identification of nonlinear structural dynamical systems," Sadhana - Academy Proceedings In Engineering Sciences, Vol. 31, 2006, pp. 399-427, Part 4.

${ }^{11}$ Guchenheimer, J. and Holmes, P., Nonlinear Oscillations, Dynamical Systems, and Bifurcation of Vector Field, Springer-Verlag, New York, 1983.

${ }^{12}$ Wiggins, S., Introduction to Applied Nonlinear Dynamical Systems and Chaos, Springer-Verlag, 1990 .

18 of 19

American Institute of Aeronautics and Astronautics 
${ }^{13}$ Mandel, J. and Beezley, J. D., "Predictor-corrector and morphing ensemble filters for the assimilation of sparse data into high-dimensional nonlinear systems," Proceedings of the IOAS-AOLS, 11th Symposium on Integrated Observing and Assimilation Systems for the Atmosphere, Oceans, and Land Surface, San Antonio, Texas, USA, 2007, p. 4.12.

${ }^{14}$ Ristic, B., Arulampalam, S., and Gordon, N., Beyond the Kalman Filter: Particle Filters for Tracking Applications, Artech House, Boston, 2004.

${ }^{15}$ Evensen, G., "The ensemble Kalman filter: theoretical formulation and practical implementation," Ocean Dynamics, Vol. 53, No. 4, 2003, pp. 343-367.

${ }^{16}$ Maruyama, G., "Continuous Markov processes and stochastic equations," Rendiconti del Circolo Matematico di Palermo, Vol. 4, 1955, pp. 48-90.

${ }^{17}$ Constantinescu, E. M., Sandu, A., Chaib, T., and Carmichael, G. R., "Ensemble-based chemical data assimilation. I: General approach," Quarterly Journal Of The Royal Meteorological Society, Vol. 133, No. 626, 2007, pp. 1229-1243.

${ }^{18}$ Message Passing Interface, http://www.mpi-forum.org/.

${ }^{19}$ Mckay, M., Beckman, R., and Conover, W., "A comparison of three methods for selecting values of input variables in the analysis of output from a computer code," Technometrics, Vol. 21, 1979, pp. 239245.

${ }^{20}$ Olsson, A. and Sandberg, G., "Latin hypercube sampling for stochastic finite element analysis," Journal of Engineering Mechanics, Vol. 128, 2002, pp. 121125. 